\title{
What is the efficacy of RTS,S?
}

\author{
Christopher J.A. Duncan ${ }^{1}$ [Research Fellow] and Adrian V.S. Hill ${ }^{1}$ [Professor] \\ ${ }^{1}$ Centre for Clinical Vaccinology and Tropical Medicine, University of Oxford, Churchill Hospital, \\ Old Road, Oxford, OX3 7LJ
}

Whitty's insightful editorial ${ }^{1}$ puts the recent phase III RTS,S malaria vaccine trial results ${ }^{2}$ in the wider context of malaria control. While vaccines are an important potential component of this effort, they are not necessarily the complete solution. RTS,S is clearly a big step in the right direction, although further advances are needed towards the goal of a highly effective malaria vaccine. ${ }^{1}$

The decision to publish interim efficacy data from an ongoing phase III study is unusual, ${ }^{3}$ and others have questioned the headline efficacy figure of around $50 \%$ in time to first malaria episode. ${ }^{4}$ Efficacy estimates will critically influence decisions on the public health role for RTS,S, and we wish to clarify some aspects of the published analysis.

A readily interpretable method of vaccine efficacy analysis involves calculating the risk ratio (the proportion of malaria in the intervention group over the control group). ${ }^{5}$ Using this approach efficacy against clinical malaria in older children is more modest at $34 \%$ (intention-to-treat) or 36\% (per-protocol).

RTS,S is thought to reduce the risk of infection from each exposure, rather than conferring "all or nothing" protection on a proportion of recipients. ${ }^{5}$ By this hypothesis, everyone vaccinated will eventually experience malaria if transmission is high enough. ${ }^{5} \mathrm{In}$ other words, the vaccine should have a greater effect on the incidence rate of the first or total episodes of clinical malaria than on the overall proportion of people experiencing it, a conclusion supported by the phase III data. ${ }^{2}$ While analysis of hazard and incidence rate ratios are completely valid ${ }^{5}$, the risk ratio for clinical malaria provides additional highly relevant information to both policy makers and parents of immunised children, and should also be reported.

\section{References}

1. Whitty CJ. The RTS,S malaria vaccine. Bmj. 2011; 343:d6986. [PubMed: 22034149]

2. First Results of Phase 3 Trial of RTS,S/AS01 Malaria Vaccine in African Children. The New England journal of medicine. 2011 [PubMed: 22007715]

3. White NJ. A Vaccine for Malaria. The New England journal of medicine. 2011 [PubMed: 22007716]

4. Butler D. Malaria vaccine results face scrutiny. Nature. 2011; 478(7370):439-40. [PubMed: 22031413]

5. Lievens M, Aponte JJ, Williamson J, Mmbando B, Mohamed A, Bejon P, et al. Statistical methodology for the evaluation of vaccine efficacy in a phase III multi-centre trial of the RTS, S/ AS01 malaria vaccine in African children. Malar J. 2011; 10:222. [PubMed: 21816030]

Conflict of Interest: AVSH is a named inventor on patent applications covering malaria vectored vaccines and immunisation regimes. 